\title{
An iterative method for optimal resolution-constrained polar quantizer design
}

\author{
Marko D. Petković ${ }^{1}$, Zoran H. Perić ${ }^{2}$, Aleksandra Ž. Jovanović ${ }^{2}$ \\ ${ }^{1}$ Faculty of Sciences and Mathematics, University of Niš, \\ Višegradska 33, 18000 Niš, Serbia \\ E-mail: dexterofnis@gmail.com \\ ${ }^{2}$ Faculty of Electronic Engineering, University of Niš, \\ Aleksandra Medvedeva 14, 18000 Niš, Serbia \\ E-mail: zoran.peric@elfak.ni.ac.yu, aleksandra.jovanovic@elfak.ni.ac.yu
}

\begin{abstract}
This paper addresses the problem of polar quantization optimization. Particularly, the aim of this investigation is to find the method for the optimal resolution-constrained polar quantizer design.

The new iterative algorithm for determination of the optimal decision and representation magnitude levels and algorithm for optimization of number of phase cells within each magnitude level, is proposed.

At high rates, the new optimal polar quantizer outperforms the optimal polar compander for $0.2 \mathrm{~dB}$, while the more significant gain should be expected at lower rates. In this paper, in order to enable practical implementation of quantizer model, algorithm which transforms real values for the optimal numbers of phase cells within magnitude levels into integer ones is also proposed. Moreover, the approximate closed form of signal to quantization ratio (SQNR) is derived.

Since circularly symmetric sources and complex presentation of signals arise in numerous applications, it can be concluded that the usage area of the suggested proposal is very wide (audio coding, image coding, spectral phase coding SPC, synthetic aperture radars systems SARs, coding of the discrete Fourier transform).

It should be emphasized that in contrast to earlier work, where models have been designed under high-rate assumption, the obtained nonuniform unrestricted polar quantizer is optimal for all rates.
\end{abstract}

\section{Introduction}

Many studies have considered the design of suboptimum vector quantizers that outperform the cartesian coordinate system quantizers, but with simpler implementation than optimal vector quantizers. In case of two-dimensional quantization of circularly symmetric densities, 
such implementation is the polar quantizer (Bucklew and Gallagher, 1979a, 1979b; Moo and Neuhoff, 1998; Pearlman, 1979; Perić and Stefanović, 2002; Swaszek, 1986; Swaszek and Ku, 1986; Swaszek and Thomas, 1983; Wilson, 1980). An intuitive reason for this superiority is that polar quantizers take advantage of the fact that circularly symmetric sources are characterized by contours of constant probability density function which are circles in the two-dimensional space (Bucklew and Gallagher, 1979a, 1979b; Jeong and Gibson, 1993; Pearlman, 1979; Perić and Stefanović, 2002; Perić et al., 2007; Swaszek and Ku, 1986; Wilson, 1980). Namely, polar quantizers have diverging angle separations, thus having small regions near the origin where the probability of vector occurrence is higher and enlarging the regions as they are removed from the origin. Hence, these schemes require the source symbols be represented in their polar form with the resulting polar coordinates processed by scalar quantizers (Bucklew and Gallagher, 1979a, 1979b; Moo and Neuhoff, 1998; Pearlman, 1979; Perić and Stefanović, 2002; Swaszek, 1986; Swaszek and Ku, 1986; Swaszek and Thomas, 1983; Wilson, 1980). According to the type of utilized scalar quantizers, there are several polar quantizer models. There are models in which uniform quantizers are applied for the phase as well as magnitude quantization (uniform polar quantizers) (Moo and Neuhoff, 1998; Perić and Stefanović, 2002; Swaszek, 1985). There are also models in which reconstruction and decision magnitude levels are not uniformly distributed i.e. nonuniform polar quantizers (Perić et al., 2007; Swaszek and Ku, 1986; Swaszek and Thomas, 1983). It should be also emphasized that Wilson (Wilson 1980) first defined the unrestricted polar quantizers, i.e. proposed a different number of level for the phase quantizers due to satisfaction of the mean square error criteria. Therefore, amplitude and phase can be quantized separately, which is in this case called strict polar quantization (SPQ) (Pearlman, 1979). They can be also quantized jointly when the phase quantization is made dependent on the amplitude. Such quantization is called unrestricted polar quantization (UPQ) (Wilson, 1980). Here can be noted that solution in (Wilson, 1980) is valid when the number of reconstruction points is small. The quantizers were derived analytically under high-rate assumptions. In (Swaszek and Thomas, 1983) the optimal nonuniform SPQ model is designed, while in (Swaszek, 1985) the idea of UPQ is realized for the large number of reconstruction points. In (Perić and Stefanović, 2002) the UPQ is optimized assuming that each scalar quantizer is a uniform one. In (Swaszek and $\mathrm{Ku}, 1986$ ) the optimal UPQ is designed under constraint that scalar compander is used for magnitude processing. In the same paper, it is also shown that obtained optimal polar compander asymptotically approaches the optimal polar quantization performance. Opposite to cited researches, in this paper, during optimal polar quantuzer designing, the only constraint is a fixed number of reconstruction points (resolution constraint).

To derive the quantizer model which is optimal for all bitrates, we consider nonuniform UPQ model which does not engage scalar compander for the nonuniform distribution of the magnitude levels. Particularly, we extend the simple iterative algorithm presented in (Perić et al., 1998) and provide new one for the determination of the optimal reconstruction and decision magnitude levels. This enables us to overreach the asymptotic performance of the optimal polar 
compander (Swaszek and $\mathrm{Ku}, 1986$ ) for $0.2 \mathrm{~dB}$. On the other side, we increase model complexity and processing delay, such that our model is more applicable for moderate and lower rates. We also provide an algorithm for the computation of optimal phase reconstruction points numbers in each magnitude region. Moreover, in order to enable simple performance calculation, we also derive the approximate expression for SQNR in closed form. The presented features assure that obtained solution should be of high significance, not only for researchers, but also for engineers. Taking into consideration that circularly symmetric sources and complex presentation of signals arise in numerous applications, it can be concluded that the usage area of this proposal is very wide.

Since short-time probability density function for speech signals is a well-modeled as Gaussian (Perić et al., 2007), polar quantization can be used for speech coding. Moreover, high performance and a simple control over perceptual effects of quantization motivate the usage of UPQ for sinusoidal audio coding (Pobloth et al., 2005; Popat and Zeger, 1992; Vafin and Kleijn, 2005). Since human eye sensitivity has circularly symmetric distribution, log-polar image sampling has been utilized recently (Boluda and Pardo, 2004; Metta et al., 2004; Shortt et al., 2006). Namely, in all applications where information is embedded in the phase or frequency of a carrier signal (for example, synthetic aperture radars systems SARs (Arslan, 2001; Perić and Jovković, 2002)), a polar analog-to-digital converter provides desirable phase information. As argued in (Pearlman and Gray, 1978), discrete Fourier transform of a fairly general data sources asymptotically leads to independent Fourier coefficients that have independent Gaussian real and imaginary parts. This means that polar quantization can be also used for compressed representation of coefficients obtained from the Fourier transform of signal (Pearlman and Gray, 1978; Pearlman, 1979). Nevertheless, taking into consideration that every source can be transformed in a Gaussian by means of properly chosen filtering technique (Popat and Zeger, 2007), polar quantizer model can also be applied to other sources. The practical significance of polar quantization is also illustrated through its involvement in two patents which are related to modulators and transmitters (Hasson and Barak, 2008; Zipper, 2008). In these patents polar quantization is used for signal constellations.

The remainder of this paper is organized as follows. In Section 2 polar quantization background is presented, while in Section 3 detailed analysis of the new iterative method for the optimal polar quantizer design is performed. Section 4 considers the iterative algorithm initialization. The achieved numerical results for bivariate Gaussian source are the topics addressed in Section 5. Finally, the summary and conclusions are provided in Section 6.

\section{Polar quantization}

We say that $\operatorname{PDF} p_{X, Y}(x, y)$ of two dimensional random variable $(X, Y)$ is circular symmetric if $p_{X, Y}(x, y)=g\left(\sqrt{x^{2}+y^{2}}\right)$ for some function $g(r)$. In other words, $p_{X, Y}$ is circular symmetric if it is a function of only the radial component $r=\sqrt{x^{2}+y^{2}}$. 
Two-dimensional vector quantizer $\mathcal{Q}$ is the function

$$
\mathcal{Q}: \mathbb{R}^{2} \rightarrow\left\{P_{1}, P_{2}, \ldots, P_{N}\right\} \subset \mathbb{R}^{2}
$$

where $S_{i}=\mathcal{Q}^{-1}\left(P_{i}\right)$ is $i$-th quantization cell and $P_{i} \in \mathbb{R}^{2}$ is $i$-th representation level (reconstruction point). Polar coordinate system $(r, \theta)$ and polar quantizers are natural for two-dimensional data with circularly symmetric density. Assume that the plane $\mathbb{R}^{2}$ is partitioned into $L$ magnitude regions by $L$ magnitude decision levels (region bounds) $0=r_{1}<r_{2}<\ldots<r_{L}<$ $r_{L+1}=+\infty$. Furthermore assume that $i$-th amplitude region is divided into $M_{i}$ phase regions where $M_{i} \geq 2$ is integer and $M_{1}+M_{2}+\ldots+M_{L}=N$. Value $M_{i}$ is called number of reconstruction points in $i$-th region. It is assumed that phase decision levels $\theta_{i, j}$ are uniform, i.e. $\theta_{i, j}=2(j-1) \pi / M_{i}$, for $j=1,2, \ldots, M_{i}$. This assumption is natural due to the circular symmetry of density function. Quantizer $\mathcal{Q}_{\text {pol }}$ is called polar quantizer if it cells $S_{i, j}, i=1,2, \ldots, L$ and $j=1,2, \ldots M_{i}$ are defined as

$$
S_{i, j}=\left\{(r, \theta) \mid r_{i} \leq r<r_{i+1}, \theta_{i, j} \leq \theta \leq \theta_{i, j+1}\right\}
$$

Assume that reconstruction points $P_{i, j}$ are given in the polar coordinates as $\left(m_{i}, \psi_{i j}\right)$, i.e. that all have the same magnitude $m_{i}$ for $j=1,2, \ldots, M_{j}$. Values $m_{i}$ and $\psi_{i, j}$ are called magnitude reconstruction level and phase reconstruction level respectively and satisfy $r_{i} \leq m_{i} \leq r_{i+1}$ and $\psi_{i, j}=(2 j-1) \pi / M_{i}$. These assumptions is also natural due to the circular symmetry of density function. In the rest of the paper we use the following vector notation: $\mathbf{r}=\left(r_{2}, r_{3}, \ldots, r_{L}\right)$, $\mathbf{m}=\left(m_{1}, m_{2}, \ldots, m_{L}\right)$ and $\mathbf{M}=\left(M_{1}, M_{2}, \ldots, M_{L}\right)$.

The quality of the quantizer $\mathcal{Q}$ is measured by distortion of resulting reproduction in comparison to the original. Mostly used measure of distortion is mean-squared error. It is defined as (Gersho and Gray, 1992)

$$
D(\mathcal{Q})=\sum_{i=1}^{N} \int_{S_{i}} d\left(P, P_{i}\right) p_{X, Y}(P) d P
$$

where $d(\cdot, \cdot)$ is an Euclidean distance. The $N$-level quantizer $\mathcal{Q}^{*}$ is sad to be optimal if for any other $N$-level quantizer $\mathcal{Q}$ there holds $D(\mathcal{Q}) \geq D\left(\mathcal{Q}^{*}\right)$. In the case of polar quantizer $\mathcal{Q}_{\text {pol }}$, distorsion is given by (Swaszek and Ku, 1986; Perić et al., 2007)

$$
D\left(\mathcal{Q}_{p o l}\right)=\sum_{i=1}^{L} \sum_{j=1}^{M_{i}} \int_{r_{i}}^{r_{i+1}} \int_{\theta_{i, j}}^{\theta_{i, j+1}}\left(r^{2}+m_{i}^{2}-2 r m_{i} \cos \left(\theta-\psi_{i, j}\right)\right) \frac{f(r)}{2 \pi} d r d \theta .
$$

where we denoted $f(r)=2 \pi r g(r)$. Similarly, we say that $\mathcal{Q}_{\text {pol }}^{*}$ is L-region optimal polar quantizer if $D\left(\mathcal{Q}_{\text {pol }}\right) \geq D\left(\mathcal{Q}_{\text {pol }}^{*}\right)$ for any other $L$-region polar quantizer $\mathcal{Q}_{\text {pol }}$ By solving the integral in (2) with respect to $\theta$ and using $\theta_{i, j}=2(j-1) \pi / M_{i}$ and $\psi_{i, j}=(2 j-1) \pi / M_{i}$ we obtain the following relation

$$
D\left(\mathcal{Q}_{p o l}\right)=D(\mathbf{r} ; \mathbf{m} ; \mathbf{M})=\sum_{i=1}^{L} \int_{r_{i}}^{r_{i+1}}\left(r^{2}+m_{i}^{2}-2 r m_{i} \operatorname{sinc}\left(\frac{\pi}{M_{i}}\right)\right) f(r) d r .
$$


Hence, the distorsion $D(\mathcal{Q})$ of the polar quantizer $\mathcal{Q}$ is represented as the function of the values $r_{i}, m_{i}$ and $M_{i}$.

In the rest of the paper we assume that $\mathcal{Q}$ is given polar quantizer whose distorsion $D(\mathcal{Q})$ is given by relation (3). Our aim is to construct an iterative method for the computation of optimal polar quantizer $\mathcal{Q}_{\text {pol }}^{*}$.

\section{Iterative method}

An iterative method for optimization of two-dimensional polar quantizer, for circularly symmetric PDF, will be described. Let

$$
F_{0}(a, b)=\int_{a}^{b} f(r) d r ; \quad F_{1}(a, b)=\int_{a}^{b} r f(r) d r .
$$

Expression (3) can be written as

$$
D\left(\mathcal{Q}_{p o l}\right)=D(\mathbf{r} ; \mathbf{m} ; \mathbf{M})=\sigma^{2}+\sum_{i=1}^{L} m_{i}^{2} F_{0}\left(r_{i}, r_{i+1}\right)-2 \sum_{i=1}^{L} m_{i} \operatorname{sinc}\left(\frac{\pi}{M_{i}}\right) F_{1}\left(r_{i}, r_{i+1}\right) .
$$

\subsection{Optimization of $r_{i}$ and $m_{i}$}

Our goal is to minimize $D(\mathbf{r} ; \mathbf{m} ; \mathbf{M})$ under the constraints $0=r_{1}<r_{2}<\ldots<r_{L}<r_{L+1}=+\infty$, $r_{i} \leq m_{i} \leq r_{i+1}, M_{i} \in \mathbb{N}, M_{1}+M_{2}+\ldots+M_{L}=N$. This is constrained, mixed non-linear optimization problem. For a fixed values of $M_{i}$, an optimal point $(\hat{\mathbf{r}}, \hat{\mathbf{m}})$ must satisfy the following conditions

$$
\begin{aligned}
\frac{\partial D}{\partial r_{i}} & =\left(m_{i-1}^{2}-m_{i}^{2}-2 m_{i-1} r_{i} \operatorname{sinc}\left(\frac{\pi}{M_{i-1}}\right)+2 m_{i} r_{i} \operatorname{sinc}\left(\frac{\pi}{M_{i}}\right)\right)=0, \\
\frac{\partial D}{\partial m_{i}} & =2 m_{i} \int_{r_{i}}^{r_{i+1}} f(r) d r-2 \operatorname{sinc}\left(\frac{\pi}{M_{i}}\right) \int_{r_{i}}^{r_{i+1}} r f(r) d r=0 .
\end{aligned}
$$

From the last equations we directly obtain

$$
\begin{aligned}
\hat{r}_{i} & =\frac{\hat{m}_{i}^{2}-\hat{m}_{i-1}^{2}}{2\left[\hat{m}_{i} \operatorname{sinc}\left(\frac{\pi}{M_{i}}\right)-\hat{m}_{i-1} \operatorname{sinc}\left(\frac{\pi}{M_{i-1}}\right)\right]}, \\
\hat{m}_{i} & =\operatorname{sinc}\left(\frac{\pi}{M_{i}}\right) \frac{F_{1}\left(\hat{r}_{i}, \hat{r}_{i+1}\right)}{F_{0}\left(\hat{r}_{i}, \hat{r}_{i+1}\right)} .
\end{aligned}
$$

Note that the condition (9) is similar to the centroid condition which is satisfied by representation levels of the optimal scalar quantizer. Also by direct calculation we find that

$$
\left.\frac{\partial^{2} D}{\partial r_{i}^{2}}\right|_{r_{i}=\hat{r}_{i}, m_{i}=\hat{m}_{i}}=\left(\hat{m}_{i}^{2}-\hat{m}_{i-1}^{2}\right) \exp \left(-\frac{\hat{r}_{i}^{2}}{8}\right)>0
$$

under the condition $m_{i}>m_{i-1}$. Since $D$ is the quadratic function of $m_{i}$ it is obvious that (9) gives its global minimum with respect to $m_{i}$. Above discussion confirms that (8) and (9) gives the global minimum of $D$, with respect to $\mathbf{r}$, if an optimal point $(\hat{\mathbf{r}}, \hat{\mathbf{m}})$ satisfies $\hat{r}_{i} \leq \hat{m}_{i} \leq \hat{r}_{i+1}$ for $i=1,2, \ldots, L$. 


\subsection{Optimization of $M_{i}$}

Now consider the minimization of $D$ with respect to $\mathbf{M}$. Recall that minimization is performed under the conditions $M_{i} \in \mathbb{N}$ and $M_{1}+M_{2}+\ldots+M_{L}=N$. Suppose that $\mathbf{r}$ is fixed and $\mathbf{m}$ is determined optimally, according to (8). Additionally suppose that $r_{i}<m_{i}<r_{i+1}$. By replacing (9) into (3) we obtain

$$
D(\mathbf{r} ; \mathbf{M})=\int_{0}^{+\infty} r^{2} f(r) d r-\sum_{i=1}^{L} A_{i} \operatorname{sinc}^{2}\left(\frac{\pi}{M_{i}}\right), \quad A_{i}=\frac{\left[F_{1}\left(\hat{r}_{i}, \hat{r}_{i+1}\right)\right]^{2}}{F_{0}\left(\hat{r}_{i}, \hat{r}_{i+1}\right)}
$$

We temporary replace the condition $M_{i} \in \mathbb{N}$ with the weaker $M_{i} \geq 1$. Since $2 \sigma^{2}=\int_{0}^{+\infty} r^{2} f(r) d r$ is constant, the optimization problem reduces to

$$
\begin{array}{ll}
\max & \sum_{i=1}^{L} A_{i} \operatorname{sinc}^{2}\left(\frac{\pi}{M_{i}}\right) \\
\text { s.t. } & \sum_{i=1}^{L} M_{i}=N \\
& M_{i} \geq 0 .
\end{array}
$$

Last optimization problem can be solved using the Lagrange multipliers technique. Hence we construct Lagrange function $J(\mathbf{M})$ as

$$
J(\mathbf{M})=\sum_{i=1}^{L} A_{i} \operatorname{sinc}^{2}\left(\frac{\pi}{M_{i}}\right)-\lambda\left(\sum_{i=1}^{L} M_{i}-N\right),
$$

and find its maximum under the conditions $M_{i} \geq 2$ (since at least two representation levels should be located in each amplitude region). By differentiating expression (13) we find the necessary conditions for the local minimum of the function $J$

$$
\frac{\partial J}{\partial M_{i}}=a\left(M_{i}\right)-A_{i} \lambda=0, \quad a(x)=\frac{x-x \cos \left(\frac{2 \pi}{x}\right)-\pi \sin \left(\frac{2 \pi}{x}\right)}{\pi^{2}} .
$$

The following lemma proves that equation (system of equations) (14) has unique solution $\hat{M}_{i}$ for the fixed value of Lagrange multiplier $\lambda$.

Lemma 1 Function $a(x)$ is monotonically decreasing, convex function on half-segment $[2,+\infty)$ i.e. $a^{\prime}(x)<0$ and $a^{\prime \prime}(x)>0$, for every $x>2$.

Proof. First note that $a(x)$ is continuously differentiable function on the half-segment $[2,+\infty)$. Its derivative is given by

$$
a^{\prime}(x)=\frac{-x^{2} \cos \left(\frac{2 \pi}{x}\right)+x^{2}-2 \pi \sin \left(\frac{2 \pi}{x}\right) x+2 \pi^{2} \cos \left(\frac{2 \pi}{x}\right)}{\pi^{2} x^{2}} .
$$

Let

$$
g(t)=a^{\prime}\left(\frac{2 \pi}{t}\right)=\frac{1}{\pi^{2}}\left[\frac{1}{2} t^{2} \cos t-t \sin t-\cos t+1\right] .
$$


We consider function $g(t)$ on the half-segment $[0, \pi)$. It is also continuously differentiable on that segment. Since $g(0)=0$ and $g^{\prime}(t)=-\frac{1}{2 \pi} t^{2} \sin t<0$ for $t \in[0, \pi)$ we conclude $g(t)<0$ for $t \in(0, \pi)$ and $g(t)$ is strictly decreasing function on the same interval. Hence $a^{\prime}(x)<0$ for $x \in[2, \pi)$ and $a^{\prime}(x)$ is strictly increasing function on the same half-interval. Last implies $a^{\prime \prime}(x)>0$ and hence $a(x)$ is convex.

Since $a(x)$ is strictly decreasing function on $[2,+\infty)$ and $\lim _{x \rightarrow+\infty} a(x)=0$, equation (14) has unique solution for the fixed value of Lagrange multiplier $\lambda$. Moreover, since

$$
\frac{\partial^{2} J}{\partial M_{i}^{2}}=a^{\prime}\left(M_{i}\right)<0, \quad \frac{\partial^{2} J}{\partial M_{i} \partial M_{j}}=0
$$

unique solution of the system (14) is the global maximum of the function $J(\mathbf{M})$ on the set $[2, \infty)^{L}$.

For a given value of Lagrange multiplier $\lambda$, denote by $\tilde{M}_{i}(\lambda)$ the unique solution of (14). Function $\tilde{M}_{i}(\lambda)$ is strictly decreasing, since $a(x)$ is strictly decreasing and obviously $A_{i} \geq 0$. Furthermore $\lim _{\lambda \rightarrow 0} \tilde{M}_{i}(\lambda)=+\infty$ since $\lim _{x \rightarrow+\infty} a(x)=0$. Condition $M_{i} \geq 2$ implies that $\tilde{M}_{i}(\lambda)$ maps the half-segment $\left(0, \frac{0.4}{\lambda}\right]$ to $[2,+\infty)$.

Since the system (14) cannot be solved analytically, we obtain the approximate analytical expression for $\tilde{M}_{i}(\lambda)$. Function $a(x)$ can be expanded into the Taylor expansion around the point $x=+\infty$ as

$$
a\left(\frac{1}{x}\right)=\frac{2 \pi^{2}}{3 x^{3}}+O\left(\frac{1}{x^{5}}\right) .
$$

By replacing (17) into (14) we obtain the following approximate solution

$$
\hat{M}_{i}(\lambda)=\sqrt[3]{\frac{2 \pi^{2}}{3 \lambda A_{i}}}
$$

The absolute difference $\left|\hat{M}_{i}(\lambda)-\tilde{M}_{i}(\lambda)\right|$ can be bounded as follows

$$
\begin{aligned}
\left|\hat{M}_{i}(\lambda)-\tilde{M}_{i}(\lambda)\right| & =\tilde{M}_{i}(\lambda)\left|1-\sqrt[3]{\frac{2 \pi^{2}}{3 a\left(\tilde{M}_{i}(\lambda)\right)}}\right| \\
& =\tilde{M}_{i}(\lambda)\left|1-\left(1+\frac{4 \pi^{2}}{45 \tilde{M}_{i}^{2}(\lambda)}+O\left(\frac{1}{\tilde{M}_{i}^{4}(\lambda)}\right)\right)\right| \\
& =\frac{4 \pi^{2}}{45 \tilde{M}_{i}^{2}(\lambda)}+O\left(\frac{1}{\tilde{M}_{i}^{3}(\lambda)}\right) .
\end{aligned}
$$

It is worth mentioning that $\left|\hat{M}_{i}(\lambda)-\tilde{M}_{i}(\lambda)\right| \rightarrow 0$ when $\tilde{M}_{i}(\lambda) \rightarrow 0$. It is also decreasing function (according to (19)) and for $\tilde{M}_{i}(\lambda)=2$ there holds $\left|\hat{M}_{i}(\lambda)-2\right|=0.532$. Above discussions shows that the absolute error of the approximation of $\tilde{M}_{i}(\lambda)$ by $\hat{M}_{i}(\lambda)$ defined by (18) is less than 1 whenever $\tilde{M}_{i}(\lambda) \geq 2$. 
The value of Lagrange multiplier can be found from the condition $\sum_{i=1}^{L} \hat{M}_{1}(\lambda)=N$. Using the relation (18) we directly found

$$
\lambda=\frac{2 \pi^{2}}{3 N^{3}}\left(\sum_{i=1}^{L} A_{i}^{-1 / 3}\right)^{3} .
$$

By replacing (20) in (18) we obtain the following approximate solution of the optimization problem (12):

$$
\hat{M}_{i}=N \frac{A_{i}^{-1 / 3}}{\sum_{j=1}^{L} A_{j}^{-1 / 3}}
$$

Optimization problem (12) is an integer programming (IP) problem, since $M_{i}$ are integers. However the solution given by $(21)$ is not an integer, in general. Hence we round $\hat{M}_{i}$ to the closest integer value, i.e. we set $M_{i}^{*}=\operatorname{round}\left(\hat{M}_{i}\right)$. However after the rounding operation, values $M_{i}^{*}$ might not satisfy the condition $\sum_{i=1}^{L} M_{i}^{*}=N$. If the sum on the left side is larger than $N$, the difference $\delta=\sum_{i=1}^{L} M_{i}^{*}=N$ is substracted from $M_{i}^{*}$ corresponding to the smallest value $A_{i}$. Similarly, if the sum is less than $N$, difference is added to the $M_{i}^{*}$ corresponding to the largest $A_{i}$.

\subsection{Algorithm for optimizing $M_{i}$}

Since the optimization problem (12) is nonlinear integer programming problem, we can apply the conventional techniques for its solving (for example, Branch and Bound method (Li and Sun, 2006)). The point $\mathbf{M}^{*}=\left(M_{1}^{*}, M_{2}^{*}, \ldots, M_{L}^{*}\right)$ can be used as a initial point. However, this approach requires the implementation of integer programming method (or using the IP solver, for example MOSEK, CPLEX, etc.). We present another method which is simple for implementation and gives the results close to the optimal.

Values $M_{i}^{*}$ can be furthermore improved by the following procedure. Pick the indices $i$ and $j$ such that $i<j$. Denote

$$
f_{i j}(x)=A_{i} \operatorname{sinc}^{2}\left(\frac{\pi}{x}\right)+A_{j} \operatorname{sinc}^{2}\left(\frac{\pi}{M_{i}^{*}+M_{j}^{*}-x}\right) .
$$

Note that $f_{i j}\left(M_{i}^{*}\right)$ is the sum of two summands corresponding to $M_{i}^{*}$ and $M_{j}^{*}$ in the objective function from the optimization problem (12). From Lemma 1 we have

$$
f_{i j}^{\prime \prime}(x)=A_{i} a^{\prime}(x)+A_{j} a^{\prime}\left(M_{i}^{*}+M_{j}^{*}-x\right)<0
$$

for every $2 \leq x \leq M_{i}^{*}+M_{j}^{*}-2$. Since $f_{i j}^{\prime \prime}(x)<0$, function $f_{i j}(x)$ has at most one global minimum in the segment $\left[2, M_{i j}-2\right]$ where $M_{i j}=M_{i}^{*}+M_{j}^{*}$. This minimum can be computed as the unique solution $\hat{x}$ of the equation $f_{i j}^{\prime}(x)=0$, if $f_{i j}^{\prime}(2) f_{i j}^{\prime}\left(M_{i j}-2\right)<0$. Else it is one of the boundary points of the segment $\left[2, M_{i j}-2\right]$ (this case never happens in practice, but it is possible theoretically). Such obtained value $\hat{x}$ is again not integer, in general and hence we 
have to apply the rounding again. However since $f_{i j}^{\prime \prime}(x)<0$, integer maximum $x^{*}$ is one of the values $\lfloor\hat{x}\rfloor$ and $\lceil\hat{x}\rceil$. Value $M_{i}^{*}$ can be used as a starting point. After the procedure value $M_{i}^{*}$ is set to $x^{*}$ and $M_{j}^{*}$ is set to $M_{i j}-x^{*}$.

Since we need an integer maximum of $f_{i j}(x)$ and $M_{i}^{*}$ is a good approximation, we can obtain $x^{*}$ simply by incrementing or decrementing $M_{i}^{*}$ while $f_{i j}$ increases. More strictly, it is realised by the following procedure:

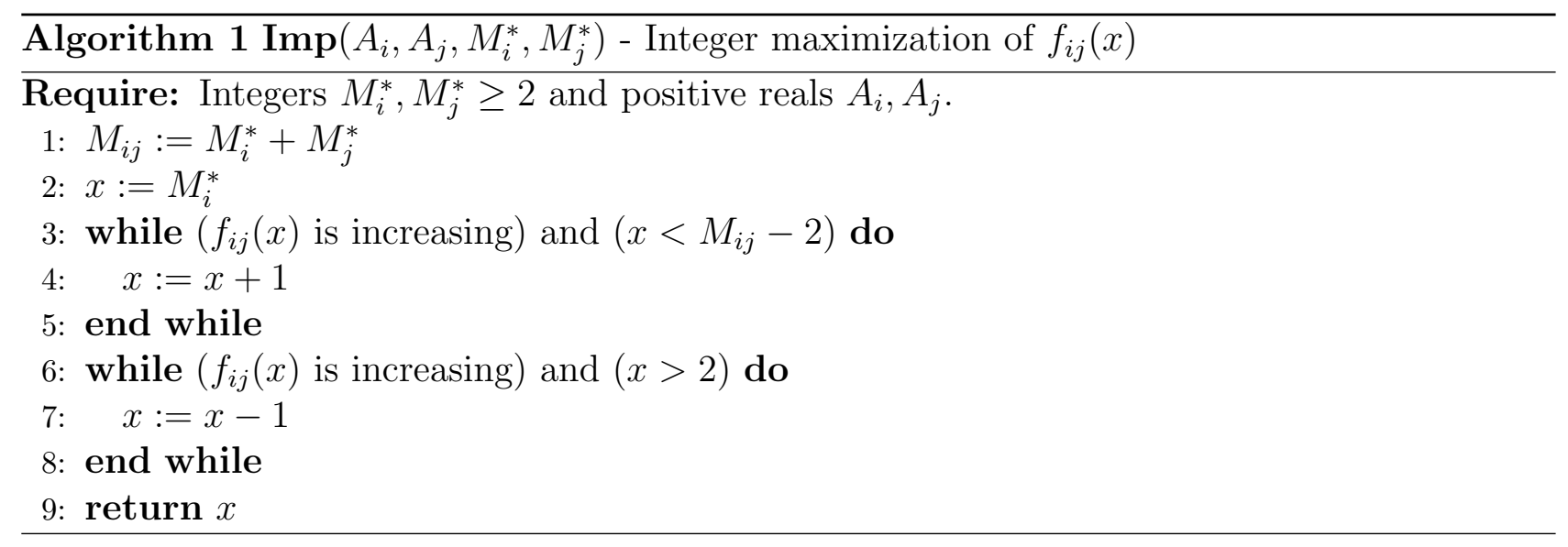

Note that exactly one of the while loops in steps 2 and 5 will be accessed. Algorithm 1 can be applied for each pair of indices $\{i, j\}$. In the practice, initial value of $M_{i}^{*}$ is usually very good approximation of the. Hence the number of steps of the Algorithm 1 is less than 3. When applied to each pair $\{i, j\}$, Algorithm 1 improves the initial point in only few number of pairs. All above discussion approves that initial values of $M_{i}^{*}$ are excellent approximation of the optimal solution of the optimization problem (12). Complete procedure is summarized in the Algorithm 2.

\subsection{Algorithm for the iterative method}

Now we are ready to formulate the complete iterative method for design of the optimal polar quantizer for circular symmetric source density. Initial values are:

1. Number of region boundaries $L$,

2. Total number of reconstruction levels $N$,

3. Initial region boundaries $0=r_{1}^{0}<r_{2}^{0}<\cdots<r_{L}^{0}<r_{L+1}^{0}=+\infty$.

In each step, algorithm first computes the new values $M_{i}^{k}$ by Algorithm 2 and known values $r_{i}^{k-1}$. Then new values $r_{i}^{k}$ are computed by relation (8) and known values $m_{i}^{k-1}$ and $M_{i}^{k}$. Finally, the new magnitude reconstruction levels $m_{i}^{k}$ are computed by relation (9) and known values $r_{i}^{k}$ and $M_{i}^{k}$. Algorithm is terminated when the relative difference between the distorsion in $k$-th and $k-1$-th iteration is less than $\epsilon$. 


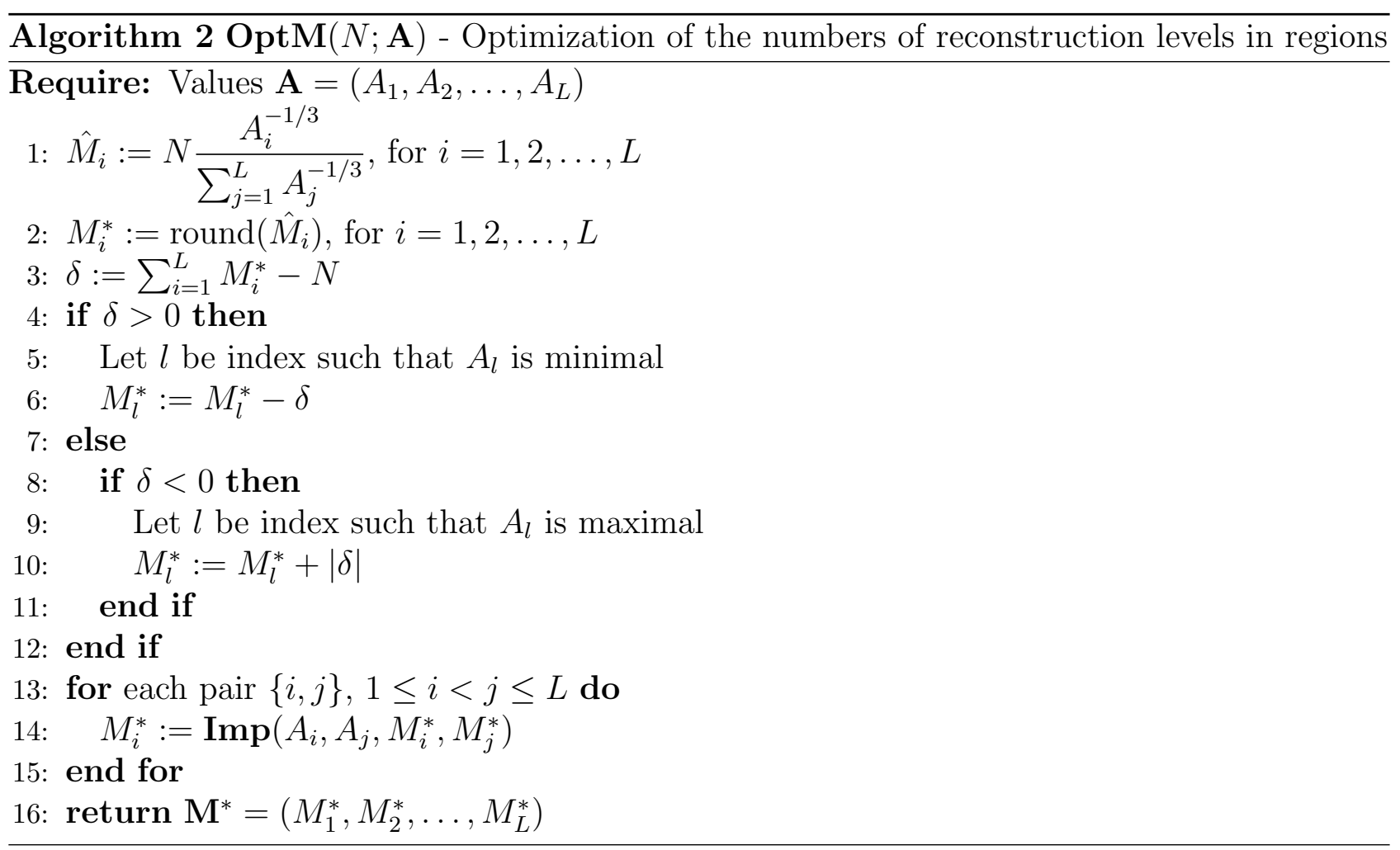

Above procedure have one drawback. It is not guaranteed that values $r_{i}^{k}$, computed by relation (9) satisfy

$$
0=r_{1}^{k}<r_{2}^{k}<\cdots<r_{L}^{k}<r_{L+1}^{k}=+\infty
$$

We say that such vector $\mathbf{r}$ is degenerate. For sufficiently high values $M_{i}^{k}$, we have $\operatorname{sinc}\left(\pi / M_{i}^{k}\right) \approx$ 1 and according to (9) holds $r_{i}^{k}=\left(m_{i-1}^{k-1}+m_{i}^{k-1}\right) / 2$. In such case, if $m_{i}^{k-1}$ are in ascending order, the same holds for $r_{i}^{k}$. So, $\mathbf{r}$ is not degenerate. But if $M_{i}^{k}$ are not sufficiently high (which is usually the case for $i=1,2$ where $M_{i}^{k}$ is around 4), relation (22) may not hold. Let $p$ be the minimal index such that $r_{p}^{k}$ violates the ascending order condition. Recall that, according to equation (10), value $r_{p}^{k}$ is global minimum of $D$ as the function of $r_{p}$, under the condition that $M_{j}=M_{j}^{k}$ are fixed, $m_{j}=m_{j}^{k-1}$ are fixed and in ascending order. Since $r_{p}^{k}<r_{p-1}^{k}, r_{p}=r_{p-1}^{k}$ is minimum of $D$, as the function $r_{p}$, under the condition $r_{p}>r_{p-1}^{k}$. Hence, $p-1$-th region (between $r_{p-1}^{k}$ and $r_{p}^{k}$ ) vanishes. Hence, the value $r_{p}^{k}$ should be dropped, $M_{p-1}^{k}$ points from $p-1$-th region should be assigned to the $p$-th region (i.e. $M_{p}^{k}:=M_{p}^{k}+M_{p-1}^{k}$ ). By repeating above procedure, we can eliminate all values $r_{i}^{k}$ violating ascending condition. Note that last procedure does not increase the value of distorsion $D$. Algorithm 3 removes the degeneracy of $\mathbf{r}$ and is based on above discussion.

Now we are ready to formulate Algorithm 4 for iterative construction of the optimal polar quantizer for circular symmetric source density.

In practice, degeneracy occurs very rarely, when the initial conditions for Algorithm 4 are suitably chosen. In next section we show one way for choosing an initial conditions and number of regions $L$. 

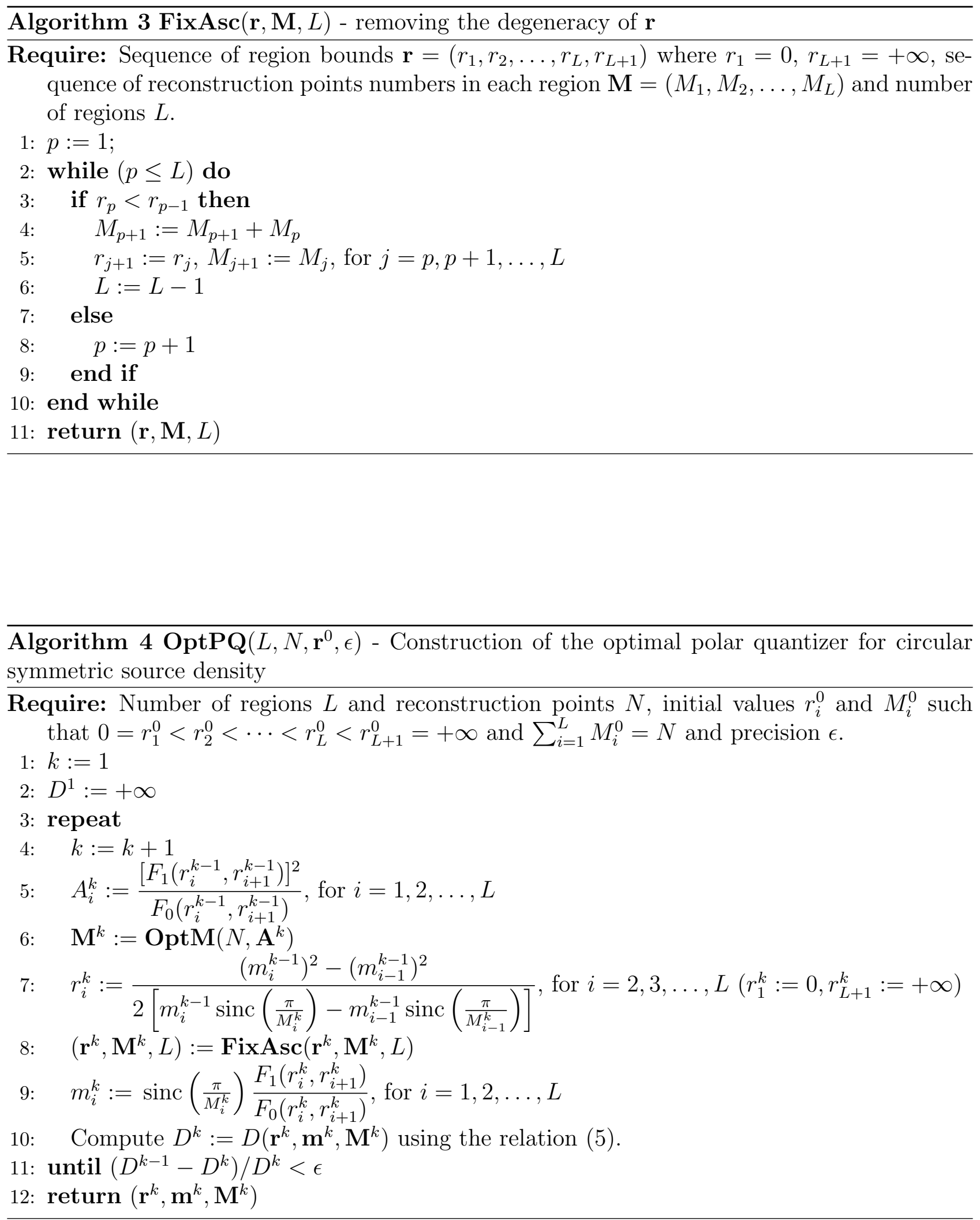
On the other side, Algorithm 4 is always convergent, since the distorsion $D^{k}$ always decreases. However, as in the case of the Lloyd-Max algorithm for scalar quantizers (Max, 1960), it is not guaranteed that the solution obtained by Algorithm 4 is optimal.

\section{Initial values}

This section provide an efficient way to choose initial values $\mathbf{r}^{0}$ and $L$ for the Algorithm 4 . It is based on the result of Swaszek and Ku (Swaszek and Ku, 1986). This approach is based on the companding technique and provide the an asymptotically optimal quantizer. Quantizers obtained using companding technique are called companding quantizers. This technique is applicable for various types of quantizers, see for example (Gersho and Gray, 1992; Jayant and Noll, 1984; Perić et al., 2007; Swaszek and Ku, 1986).

An companding polar quantizer $\mathcal{Q}_{\text {pol,com }}(x)$ is defined as $\mathcal{Q}_{\text {pol,com }}(x)=G^{-1}\left(\mathcal{U} \mathcal{Q}_{\text {pol }}(G(x))\right)$ where $\mathcal{U} \mathcal{Q}_{\text {pol }}(x)$ is an uniform polar quantizer and $G(x)$ is compressor function. It is defined by

$$
G: \mathbb{R}^{2} \mapsto B(\mathbf{0}, 1), \quad G(x)=\frac{g(r)}{r} x, \quad r=\|x\| .
$$

where $g: \mathbb{R} \mapsto[0,1]$ is polar compressor function and $B(\mathbf{0}, 1)$ is unit ball. The region bounds $y_{i}$ and magnitude representation levels $z_{i}$ of the $L$-region uniform polar quantizer $\mathcal{U} \mathcal{Q}_{\text {pol }}(x)$ are given respectively by

$$
y_{i}=\frac{i-1}{L}, i=1,2, \ldots, L+1, \quad z_{i}=\frac{y_{i}+y_{i+1}}{2}=\frac{2 i-1}{2 L}, i=1,2, \ldots, L .
$$

Region bounds $r_{i}$ an magnitude representation levels $m_{i}$ of companding polar quantizer $\mathcal{Q}_{\text {pol,com }}(x)$ are given by $r_{i}=g^{-1}\left(y_{i}\right)$ and $m_{i}=g^{-1}\left(z_{i}\right)$.

Swaszek and $\mathrm{Ku}$ considered an optimization of the companding polar quantizer $\mathcal{Q}_{\text {pol,com }}(x)$. According to (Swaszek and Ku, 1986), optimal number of regions $L$ and number of reconstruction points in $i$-th region are given by

$$
\begin{aligned}
L & =\operatorname{round}\left[\frac{\sqrt{N}}{\sqrt{2 \pi}} \frac{\int_{0}^{+\infty} x^{-1 / 4} f^{1 / 4}(x) d x}{\left[\int_{0}^{+\infty} x^{-1 / 2} f^{1 / 2}(x) d x\right]^{1 / 2}}\right] \\
M_{i}^{0} & =\frac{\sqrt{2 \pi N} f^{1 / 4}\left(m_{i}\right) m_{i}^{3 / 4}}{\left[\int_{0}^{+\infty} x^{-1 / 2} f^{1 / 2}(x) d x\right]^{1 / 2}},
\end{aligned}
$$

while an optimal polar compressor function $g(r)$ is defined by

$$
g(r)=\frac{\int_{0}^{r} x^{-1 / 4} f^{1 / 4}(x) d x}{\int_{0}^{+\infty} x^{-1 / 4} f^{1 / 4}(x) d x}
$$

Recall that $r_{i}=g^{-1}\left(y_{i}\right)$ and $m_{i}=g^{-1}\left(z_{i}\right)$. Since $L$ is integer, it is given as the integer closest to the expression in (24). However, it should be checked also values $L-1$ and $L+1$. 
Since the optimal companding polar quantizer is asymptotically optimal polar quantizer (Swaszek and $\mathrm{Ku}, 1986$ ), it can be used for the start of Algorithm 4. In other words, initial parameters of Algorithm 4 can be chosen as the corresponding parameters of optimal companding polar quantizer. In such way, number of regions should be chosen according to (24) (also values $L-1$ and $L+1$ should be tried) and initial region bounds $r_{i}^{0}=g^{-1}\left(y_{i}\right)$ where $g(r)$ is given by (26).

\section{Two-dimensional Gaussian source and numerical examples}

We test our algorithms on designing the optimal polar quantizer for two-dimensional Gaussian source. Let $(X, Y)$ be two-dimensional Gaussian random variable such that $X$ and $Y$ are uncorrelated. PDF function $p_{X, Y}(x, y)$ and function $f(r)$ are given by

$$
p_{X, Y}(x, y)=\frac{1}{2 \pi \sigma} \exp \left(-\frac{x^{2}+y^{2}}{2 \sigma^{2}}\right), \quad f(r)=r \exp \left(-\frac{r^{2}}{2}\right) .
$$

By direct computation using (4) we find

$$
\begin{aligned}
& F_{1}(a, b)=\exp \left(-\frac{a^{2}}{2}\right)-\exp \left(-\frac{b^{2}}{2}\right) \\
& F_{2}(a, b)=a \exp \left(-\frac{a^{2}}{2}\right)-\sqrt{\frac{\pi}{2}} \operatorname{erf}\left(\frac{a}{\sqrt{2}}\right)-b \exp \left(-\frac{b^{2}}{2}\right)+\sqrt{\frac{\pi}{2}} \operatorname{erf}\left(\frac{b}{\sqrt{2}}\right)
\end{aligned}
$$

where $\operatorname{erf}(x)=2 \pi^{-1 / 2} \int_{0}^{x} \exp \left(-t^{2}\right) d t$ is an error function.

All algorithms are implemented in the symbolic programming package Mathematica (version 7.0). We show some results obtained by testing our implementations for uncorrelated twovariable Gaussian source with unit variance $(\sigma=1)$. Parameters of the optimal polar quantizer for $N=64$ levels are given by

$$
\begin{aligned}
L & =6 \\
\mathbf{r} & =(0,0.276903,0.663063,1.11976,1.65484,2.34537,+\infty) \\
\mathbf{m} & =(0.11707,0.46865,0.883483,1.36178,1.93031,2.69542) \\
\mathbf{M} & =(2,6,11,15,16,14) \\
D & =0.0620599, \quad \text { SQNR }=15.0822 \mathrm{~dB} .
\end{aligned}
$$

Also, parameters of the optimal polar quantizer for $N=128$ levels are given by

$$
\begin{aligned}
L & =8 \\
\mathbf{r} & =(0,0.323755,0.62253,0.943175,1.29048,1.68843,2.16081,2.80557,+\infty) \\
\mathbf{m} & =(0.193301,0.475613,0.780552,1.10901,1.47377,1.89305,2.4061,3.10778) \\
\mathbf{M} & =(4,9,14,18,22,23,22,16) \\
D & =0.0317631, \quad \mathrm{SQNR}=17.9911 \mathrm{~dB} .
\end{aligned}
$$




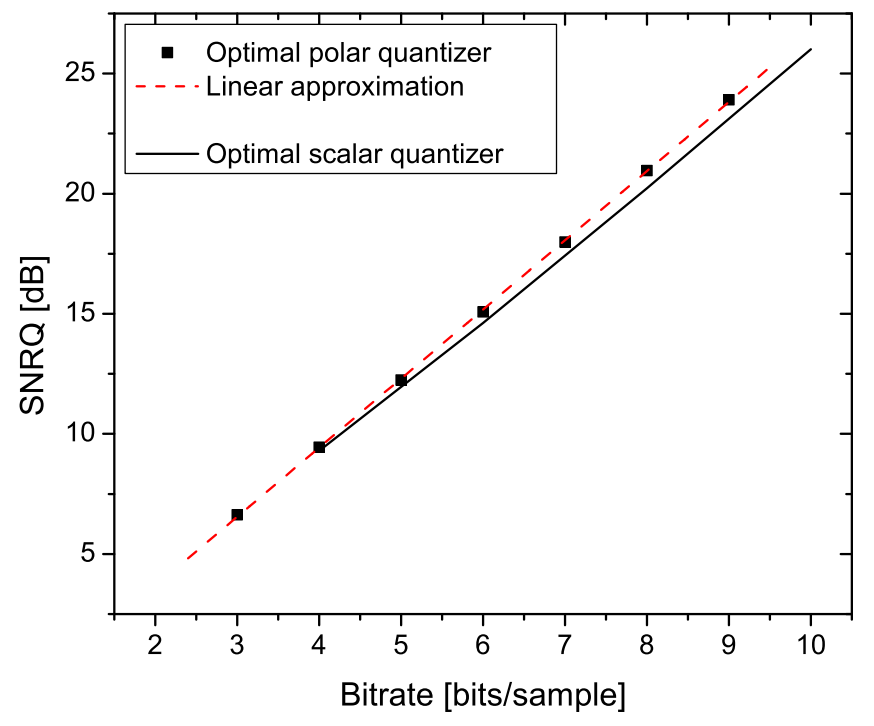

Figure 1: SQNR value of optimal polar quantizer and optimal scalar quantizer for different values of bitrate $R$.

Here $D$ denotes the distorsion of quantizer, defined by (3) and SQNR $=10 \log \left(2 \sigma^{2} / D\right)$ denotes the value of Signal-to-Quantizer-Noise-Ratio. Figure 1 shows the dependence of SQNR of the optimal polar quantizer versus the total bitrate $R=\log _{2} N$. We also included, for the purpose of comparation, the dependence of SQNR for the optimal scalar quantizer (Jayant and Noll, 1984).

It can be noticed that the dependence is almost linear. Asymptotic expression for the distorsion $D$, valid for large values of $N$, is equal to $D=\frac{2 \pi}{3 N}$ (Perić, et al., 2007; Swaszek and $\mathrm{Ku}, 1986)$. Therefore, the asymptotic dependence of SQNR, as a function of $R$ is also linear. By linear regression we obtain the approximate values of the parameters of linear dependence SQNR $\approx-2.09141+2.87887 \cdot R$, from Figure 1, where the correlation coefficient equal to 0.99992. This confirms the linear dependence of SQNR for smaller values of bitrate $R$.

Note that for $N=256$, the distorsion and SQNR of the optimal polar quantizer are given by $D=0.0159589$ and SQNR $=20.9072 \mathrm{~dB}$. Optimal uniform polar quantizer (UPQ) (Swaszek and $\mathrm{Ku}, 1986$ ) has total distorsion $D_{U Q}=0.01683$ and $\mathrm{SQNR}=20.7495 \mathrm{~dB}$ while an optimal two-dimensional vector quantizer has $D_{V Q}=0.01575$ and $\mathrm{SQNR}=21.0375 \mathrm{~dB}$ (Gersho and Gray, 1992). Hence the difference in SQNR values between an optimal vector and optimal polar quantizer is only $0.057224 \mathrm{~dB}$ while the difference between an optimal vector and optimal uniform polar quantizer is $0.28 \mathrm{~dB}$.

Number of iterations required for our method (Algorithm 4), for $\epsilon=10^{-6}$ is 244. It shows the slow convergence of our method. 


\section{Conclusion}

In this paper we present one new method for the resolution-constrained polar quantization optimization. We provide iterative algorithm for determination of the optimal reconstruction and decision magnitude levels, as well as, algorithm for optimization of number of phase cells within each magnitude level. We point out that firstly we obtain real values for the optimal numbers of reconstruction points in magnitude regions and after that we assure algorithm which enables transformation of optimal real values to the optimal integer ones.

The concept of proposed design method can be also considered as the iterative improving of the optimal polar compander. The achieved gain in reproduced signal quality is not smaller than $0.2 \mathrm{~dB}$, while design and implementation complexities are enlarged. Because of that, the obtained optimal polar quantizer can be used for moderate and lower rates in analogto-digital conversion of signals with circularly symmetric densities and complex presentation (audio coding, image coding, spectral phase coding SPC, synthetic aperture radars systems SARs, coding of the discrete Fourier transform). Furthermore, a possibility that any kind of density can be transformed in a Gaussian distribution by means of properly chosen filtering gives additional importance to the proposed quantizer model.

In this paper we also derive the approximate expression for SQNR in closed form which enables easily calculation of optimal polar quantizer performances. Therefore, we believe that the novel quantizer model is of high significance not only for researchers but also for engineers.

\section{Acknowledgement}

Authors wishes to thank to anonymous referees for useful comments improving the quality of the paper.

\section{References}

[1] Arslan F.T. (2001), Adaptive bit rate allocation in compression of SAR images with JPEG2000, M. S. thesis, University of Arizona, USA.

[2] Boluda F. and Pardo F. (2004), Space variant image processing: taking advantage of data reduction and polar coordinates, Electronic Imaging, vol. 14, no.1, pp. 1-12.

[3] Bucklew J.A. and Gallagher N.C. (1979), Quantization schemes for bivariate Gaussian random variables, IEEE Transaction on Information Theory, vol. IT-25, pp. 537-543.

[4] Bucklew J.A. and Gallagher N.C. (1979), Two-dimensional quantization of bivariate circularly symmetric densities, IEEE Transaction on Information Theory, vol. IT-25, pp. 667-671.

[5] Gersho A. and Gray R. (1992), Vector Quantization and Signal Compression, Boston, Dordrecht, London: Kluwer Academic Publishers.

[6] Hasson J. and Barak I. (2008), Transmitter, Patent No. US 7,336,753 B2.

[7] Jayant N.S. and Noll P. (1984), Digital coding of waveforms, Principles and Applications to Speech and Video, Prentice-Hall, New Jersey. 
[8] Jeong D. and Gibson J. (1993), Uniform and piecewise uniform lattice vector quantization for memoryless Gaussian and Laplacian sources, IEEE Transaction on Information Theory, vol. 39, no. 3, pp. 786-804.

[9] Li D. and Sun X. (2006), Nonlinear integer programming, Springer, Boston.

[10] Max J. (1960), Quantizing for minimum distortion, IRE, Transactions on Information Theory, Vol. IT-6, pp. 7-12.

[11] Metta G., Gasteratos A. and Sandini G. (2004), Learning to track colored objects with log-polar vision, Mechatronics, vol. 14, pp. 989-1006.

[12] Moo P.W. and Neuhoff D.L. (1998), Uniform polar quantization revisited, In Proc. IEEE International Symposium on Information Theory ISIT'98, pp. 100, Cambridge, USA.

[13] Pearlman W.A. (1979), Polar quantization of a complex Gaussian random variable, IEEE Transactions on Communications, vol. COM-27, pp. 892-899.

[14] Pearlman W.A. and Gray R.M. (1978), Source coding of the discrete Fourier transform, IEEE Transaction on Information Theory, vol. IT-24, no.6, pp.683-692.

[15] Perić Z.H. and Jovković J.D. (2002), Application of the optimal uniform polar quantization on complex reflectivity function, Advances in Electrical and Computer Engineering, vol. 9, no.1, pp. 80-96.

[16] Perić Z.H. and Stefanović M.Č. (2002), Asymptotic analysis of optimal uniform polar quantization, International Journal of Electronics and Communications (AEU), vol. 56, no.5, pp. 345-347.

[17] Perić Z.H., Jovanović A.Ž., Stefanović M.Č. and Bogosavljević S.M. (2007), Switched nonuniform polar quantization of sources with a side dynamic range of power, Journal of Communications Technology and Electronics, vol. 52, no. 12, pp. 1340-1349.

[18] Perić Z.H., Djordjević I.B., Bogosavljević S.M. and Stefanović M. Č. (1998), Design of signal constellations for Gaussian channel by using iterative polar quantization, In Proc. 9th Mediterranean Electrotechnical Conference MELECON 98, vol. 2, pp. 866-869, Tel-Aviv, Israel.

[19] Pobloth H., Vafin R. and Kleijn B. (2005), Multivariate block polar quantization, IEEE Transactions on Communications, vol. 53, no. 12.

[20] Popat K. and Zeger K. (1992), Robust quantization of memoryless sources using dispersive FIR filters, IEEE Trans.Commun., vol. 40, pp. 1670-1674.

[21] Ravelli E. and Daudet L. (2007), Embedded polar quantization, IEEE Signal Processing Letters, vol.14, no. 10 .

[22] Shortt A., Naughton T. and Javidi B. (2006), A companding approach for nonuniform quantization of digital holograms of three-dimensional objects, Optics Express, vol.14, no. 12, pp. 5129-5134.

[23] Swaszek P.F. (1985), Uniform spherical coordinate quantization of spherically symmetric sources, IEEE Transactions on Communications, vol. COM-33, no.6.

[24] Swaszek P.F. and Ku T.W. (1986), Asymptotic Performance of Unrestricted Polar Quantizer, IEEE Transactions on Information Theory, vol. 32, pp. 330-333.

[25] Swaszek P.F. and Thomas J.B. (1983), Multidimensional spherical coordinate quantization, IEEE Transaction on Information Theory, vol. IT-29, no.4.

[26] Vafin R. and Kleijn B. (2005), Entropy-constrained polar quantization and its application to audio coding, IEEE Transaction on Speech and Audio Processing, vol. 13, no.2.

[27] Wilson S.G. (1980), Magnitude phase quantization of independent Gaussian variates, IEEE Transactions on Communications, vol. COM-28, pp. 1924-1929. 
[28] Zipper E. (2008), Outphasing modulators and methods of outphasing modulation, Patent No. US $7,356,315$ B2.

Zoran H. Perić was born in Niš, Serbia, in 1964. He received the B. Sc. degree in electronics and telecommunications from the Faculty of Electronic Engineering, Niš, Serbia, Yugoslavia, in 1989, and M. Sc. degree in telecommunications from the University of Niš, in 1994. He received the $\mathrm{Ph}$. D. degree from the University of Niš, also, in 1999. He is currently Professor at the Department of Telecommunications and vicedean of the Faculty of Electronic Engineering, University of Niš, Serbia. His current research interests include the information theory, source and channel coding and signal processing. He is particularly working on scalar and vector quantization techniques in speech and image coding. He was author and coauthor in over 140 papers in digital communications. Dr Perić has been a Reviewer for: IEEE Transactions on Information Theory, Informatica, COMPEL. He is member of Editorial Board of Journal "Electronics and Electrical Engineering".

Marko D. Petković was born in Niš, Serbia, in 1984. He graduated mathematics and computer science at the Faculty of Sciences and Mathematics, Niš, Serbia in 2006, and telecommunications at Faculty of Electronic Engineering, Niš, Serbia in 2007. He received Ph.D. degree in computer science from University of Niš in 2008. Currently he works as assistant professor at the Faculty of Sciences and Mathematics, Niš, Serbia. His research interests include the source and channel coding, generalized inverses of matrices, Hankel determinants and optimization methods. He is the author of about 30 papers (13 of them in peer-reviewed international journals). Dr Petković has been Reviewer for Journal of Computational and Applied Mathematics, Computers and Mathematics With Applications and International Journal of Computer Mathematics. He is supported by Ministry of Science, Republic of Serbia, Grant No. 144011.

Aleksandra Ž. Jovanović was born in Niš, Serbia in 1971. She received the B.Sc., M.Sc. and Ph.D degrees in electrical engineering from the Faculty of Electronic Engineering (Department of Telecommunications), University of Niš, Serbia, in 1995, 1999 and 2007, respectively. Her field of interest includes digital telecommunication, information theory and source coding. She has authored and coauthored over 50 scientific papers. 